TITLE:

\title{
Atomic alkali-metal gas cells at liquid-helium temperatures: Loading by light-induced atom desorption
}

\section{AUTHOR(S):}

Hatakeyama, A; Enomoto, K; Sugimoto, N; Yabuzaki, T

\section{CITATION:}

Hatakeyama, A ... [et al]. Atomic alkali-metal gas cells at liquid-helium temperatures: Loading by light-induced atom desorption. PHYSICAL REVIEW A 2002, 65(2): 022904.

ISSUE DATE:

2002-02

URL:

http://hdl.handle.net/2433/50030

RIGHT:

Copyright 2002 American Physical Society 


\title{
Atomic alkali-metal gas cells at liquid-helium temperatures: Loading by light-induced atom desorption
}

\author{
A. Hatakeyama, K. Enomoto, N. Sugimoto, and T. Yabuzaki* \\ Department of Physics, Faculty of Science, Kyoto University, Kyoto 606-8502, Japan
}

(Received 19 December 2000; revised manuscript received 23 March 2001; published 16 January 2002)

\begin{abstract}
We have studied the loading of gaseous alkali-metal atoms into glass cells held at about $2 \mathrm{~K}$ by light-induced atom desorption (LIAD). In LIAD loading, the cell containing dense helium gas and a small amount of alkali metal is irradiated with cw laser light. This technique has two unique features to be investigated: alkali-metal atoms are desorbed, even by weak laser irradiation, from the cell walls not covered visibly with alkali metal, and loading efficiency is much higher below the superfluid transition temperature for the helium inside the cell than above this temperature. On the basis of extensive experimental studies for Rb presented in this paper, we give a consistent picture of the loading mechanism: alkali-metal atoms are optically desorbed from alkali-metal particles on the walls, then transported effectively into the cell volume by helium-gas flow produced by the continuous evaporation of the helium film, which is supplied by the superfluid-film flow on the walls from the bulk liquid helium at the bottom of the cell.
\end{abstract}

DOI: 10.1103/PhysRevA.65.022904

PACS number(s): 34.50.Dy, 36.40.-c, 67.70. $+\mathrm{n}$

\section{INTRODUCTION}

The utilization of cold helium allows novel experimental environments at low temperatures for atomic and molecular physics [1]. Fascinating features of these cold-helium environments are not only the capability of cooling a variety of sample species, but also the weak perturbation on embedded atoms and molecules and their own unique properties such as superfluidity. Some examples illustrating these features are as follows: the helium-buffer-gas loading of magnetic traps has made it possible to cool and trap many kinds of atoms and molecules to which laser-cooling techniques are not applicable [2]; helium-cluster isolation spectroscopy has provided rotationally resolved spectra of molecules inside helium clusters [3] and has been used to investigate superfluidity in the finite quantum system [4]; spin-related physics experiments have been performed by exploiting the inertness of cold helium with respect to spin relaxation of sample species [5].

In a previous publication [6] we have reported another interesting application of cold helium: atomic $\mathrm{Rb}$ gas cells held at about $2 \mathrm{~K}$, containing helium buffer gas in saturated equilibrium with a superfluid helium film coating the cell walls. An atomic $\mathrm{Rb}$ gas with a density of the order of $10^{8} \mathrm{~cm}^{-3}$ loaded simply by $\mathrm{cw}$ laser irradiation of the cell resides in the cold cell for approximately $10 \mathrm{~s}$ at $1.85 \mathrm{~K}$, enabling a new class of atomic physics experiments in helium buffer gas at low temperatures. Among them, experiments related to extremely slow spin relaxation for sample species in cold helium gas would be most promising. This slow spin relaxation was demonstrated for optically polarized $\mathrm{Rb}$ atoms, whose electronic spin relaxation time for processes (such as Rb-He collisions) that do not cause the loss of the atomic $\mathrm{Rb}$ was found to be much longer than the lifetime $(\sim 10 \mathrm{~s})$ of the gaseous $\mathrm{Rb}$ atoms in the cold cell. It

*Email address: yabuzaki@scphys.kyoto-u.ac.jp is worth emphasizing that the slow spin relaxation in cold helium gas is an excellent example of the weak perturbation on sample species by cold helium.

In the experiment mentioned above, the loading of $\mathrm{Rb}$ atoms into the cell by light-induced atom desorption (LIAD) was introduced as a key technique, although the physical mechanism of LIAD loading remained to be investigated. To our knowledge, this was the first observation of LIAD at such low temperatures as about $2 \mathrm{~K}$. Furthermore, LIAD loading has two unique features: by weak cw laser irradiation, a substantial number of $\mathrm{Rb}$ atoms are desorbed not from $\mathrm{Rb}$ metal, such as Rb films or droplets in the cell, but from transparent glass walls that are not covered visibly with $\mathrm{Rb}$ metal; LIAD loading is considerably effective only when helium gas in the cell liquifies (below $\sim 1.9 \mathrm{~K}$ in the previous paper).

In this paper we present a systematic investigation of the cell-loading mechanism. We measured the dependences of the number of loaded $\mathrm{Rb}$ atoms on the superfluidity of helium in the cell, on the repetitions of loading, on the power and the wavelength of the loading laser light, and on the amount of $\mathrm{Rb}$ deposited on the wall surfaces. The obtained experimental results indicate a consistent picture of the loading mechanism: $\mathrm{Rb}$ atoms are desorbed from $\mathrm{Rb}$ particles on the cell walls irradiated with laser light. In addition, this laser light warms the cell walls and causes the continuous evaporation of the helium film, which is supplied by the superfluid flow on the walls, and subsequently produces helium-gas flows. By these superfluid-driven gas flows, the desorbed Rb atoms are effectively transported from the vicinity of the cold walls into the cell volume through a dense helium gas, while this dense helium gas serves to confine the embedded $\mathrm{Rb}$ atoms for a long time. Accordingly, we can say that the cold atomic gas cell also owes its realization by LIAD loading to one of the unique features of the cold-helium environments, namely, superfluidity.

We add to the concluding section some experimental results on LIAD loading for other alkali metals (K, Cs) and on the conventional laser ablation loading we also attempted. 


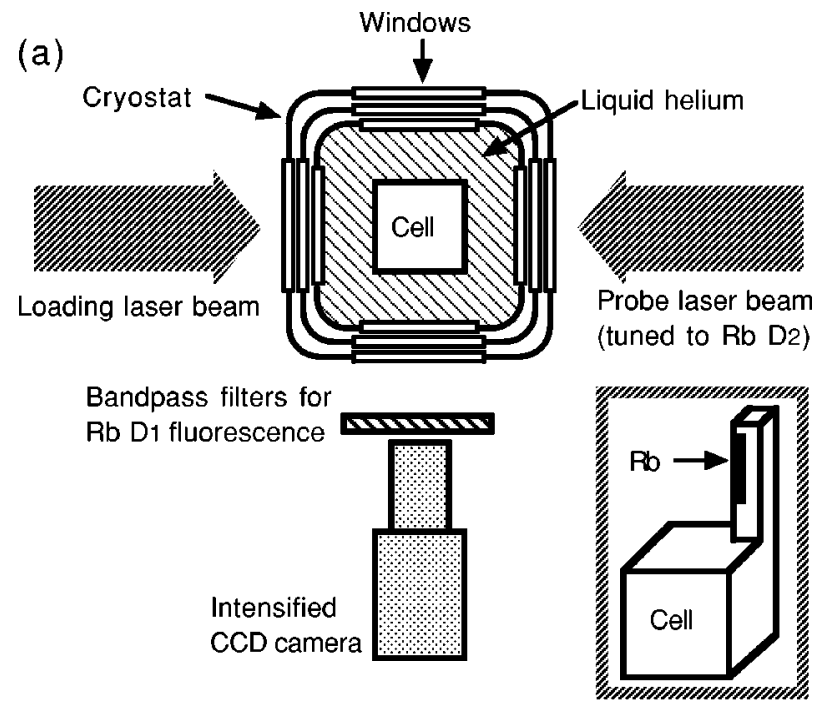

(b)

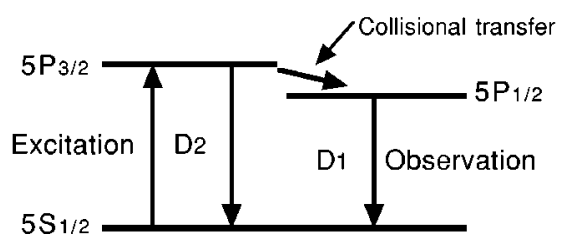

FIG. 1. (a) Experimental apparatus (top view). A cubic cell (see the inset) is placed in liquid helium in a cryostat. Rb atoms introduced into the cell with a loading beam are excited with a probe beam tuned to the $D_{2}$ transition, and the $D_{1}$ fluorescence is detected with an intensified CCD camera. (b) Energy-level diagram of Rb relevant to the observation of $\mathrm{Rb}$ atoms.

\section{EXPERIMENT}

We describe in this section the fundamental procedure of the experiment, that is, how to prepare sample cells and how to load and observe $\mathrm{Rb}$ atoms in the cell cooled to liquidhelium temperatures. The experimental apparatus is sketched in Fig. 1(a).

Cubic sample cells (2-cm inner sides) made of Pyrex glass (1 mm thickness) were filled as follows. The cell was attached to a glass vacuum system containing a $\mathrm{Rb}$ ampoule and connected to a ${ }^{4} \mathrm{He}$ gas cylinder. The cell was evacuated to of the order of $10^{-6}$ torr and outgassed at about $500 \mathrm{~K}$ for a day. $\mathrm{Rb}$ metal was then transferred into the cell through evacuated glass tubes by evaporation and recondensation using a flame of a torch. The $\mathrm{Rb}$ metal was accumulated mainly in the capillary [see the inset in Fig. 1(a)] connecting the cubic body of the cell to the evacuated glass tubes. During this procedure some $\mathrm{Rb}$ metal was introduced also into the cubic body of the cell. As described in Sec. III, the amount of $\mathrm{Rb}$ deposited on the cell walls affected the efficiency of LIAD loading. Therefore this amount must be controlled by introducing more $\mathrm{Rb}$ metal into the cell or driving excess $\mathrm{Rb}$ metal out of the cell with a torch. In preparation of typical cells used in the experiment, a small amount of $\mathrm{Rb}$ metal was introduced once so that $\mathrm{Rb}$ films were formed on the walls. Then these films were gradually removed by heating the cell with the torch just until the films became invisible with the naked eye. If there were $\mathrm{Rb}$ droplets in the cell, they were also driven out. We describe in Sec. III how the amount of $\mathrm{Rb}$ deposited on the walls affects loading efficiency. After the amount of $\mathrm{Rb}$ was controlled, ${ }^{4} \mathrm{He}$ gas (purity 99.999\%) was introduced through a liquid- $\mathrm{N}_{2}$-cooled trap for further purification. When the cell was sealed off at the capillary, its cubic body was dipped in liquid $\mathrm{N}_{2}(77 \mathrm{~K})$ to increase the helium gas density, with the helium pressure being kept below $1 \mathrm{~atm}$ (about 730 torr). The helium gas in the cell filled in this way had a density of about $9 \times 10^{19} \mathrm{~cm}^{-3}$ and therefore began liquifying at $T_{c} \simeq 1.9 \mathrm{~K}$, where its density reached the saturated vapor density. The superfluid transition of helium in the cell also occurs at almost the same temperature [7].

The sealed cells mentioned above were used for most of the measurements presented here, and they allowed repeated experiments under the same sample conditions. In addition to the sealed cells we made a density-controllable cell. The cubic body of this cell was connected to glass and metal tubes, through which more helium gas could be introduced into the cell to obtain higher liquifying temperatures than in the case of the sealed cells.

The cell was mounted in the sample chamber of a pumped liquid-helium cryostat with optical windows. At liquidhelium temperatures, the cell was immersed in liquid helium to minimize the heating of the cell by laser beams directed through the cell. In particular, superfluid helium can effectively dissipate the applied heat. An atomic $\mathrm{Rb}$ gas was loaded into the cell by directing a $\mathrm{cw}$ laser beam (a loading beam) through the whole volume of the cell. To observe $\mathrm{Rb}$ atoms loaded into the cell, they were excited by a probe beam tuned to the $5 S_{1 / 2} \rightarrow 5 P_{3 / 2} D_{2}$ transition (wavelength $780 \mathrm{~nm}$ ). Then we monitored the $D_{1}$ fluorescence at $795 \mathrm{~nm}$ from the $5 P_{1 / 2}$ state populated by collisions between $\mathrm{Rb}$ atoms in the $5 P_{3 / 2}$ state and helium gas [see Fig. 1(b)]. The $D_{1}$ fluorescence was detected with an intensified chargecoupled-device (ICCD) camera through bandpass filters to suppress the large backgrounds due to the scattered laser radiation. The images recorded with the ICCD camera provided information about the two-dimensional spatial distribution of the density of the $\mathrm{Rb}$ atoms averaged along the direction of the camera.

The atomic density of the Rb gas in the cell was roughly estimated by comparing the intensity of the $D_{1}$ fluorescence observed at low temperatures and that observed for a saturated $\mathrm{Rb}$ vapor (whose density is known [8]) at room temperature in the cell containing plenty of Rb metal. This estimation required the knowledge of the $D_{2}$ excitation rate and of the branching ratio for the $D_{1}$ fluorescence, which were varied with temperature. The $D_{2}$ excitation rate was estimated from the pressure-broadened excitation line profile using the relation $\int \sigma(\nu) d \nu=\pi r_{0} c f$, where $\sigma(\nu)$ is the photonabsorption cross section at a frequency $\nu, r_{0}=2.82$ $\times 10^{-13} \mathrm{~cm}$ is the classical electron radius, $c$ is the velocity of light, and $f$ is the transition oscillator strength. The measured $D_{2}$ linewidths (full width at half maximum) were, for example, $\sim 60 \mathrm{GHz}$ at $293 \mathrm{~K}$ and $\sim 12 \mathrm{GHz}$ at $1.85 \mathrm{~K}$ [6]. The $D_{1}$ branching ratio was measured by observing the $D_{1}$ and $D_{2}$ fluorescence simultaneously with a spectrometer coupled to a CCD detector [6]. The measured ratios were, for 
example, $\sim 0.5$ at $293 \mathrm{~K}$ and $\sim 0.9$ at $1.85 \mathrm{~K}$. In the temperature range from 1.7 to $2.0 \mathrm{~K}$, where we performed experiments, the $D_{1}$ fluorescence efficiency (the $D_{2}$ excitation rate times the $D_{1}$ branching ratio) was about ten times higher than at room temperature. Therefore the $\mathrm{Rb}$ density in this temperature range was about ten times lower than at room temperature when the intensities of the $D_{1}$ fluorescence are the same. Detailed data required to determine the density of $\mathrm{Rb}$ atoms will be presented in our following publication on the properties of atomic alkali-metal gases loaded in the cold cells.

Finally in this section we comment on the power of the laser beam and the temperature of the cell. Since laser beams passed through three cryostat windows (two of which were antireflection coated) before striking the cell, the beam power applied to the cell was about $90 \%$ of the power outside the cryostat. The values of the beam power mentioned in this paper are those actually applied to the cell.

These laser beams warmed up the cell. During the typical loading by irradiation with a laser beam of power $180 \mathrm{~mW}$ [9] for $10 \mathrm{~s}$ the temperature inside the cell rose by a few tens of $\mathrm{mK}$. This temperature increase was inferred from an increase of the saturated helium vapor pressure in the cell, which was measured with a pressure gauge attached to the density-controllable cell or estimated by measuring the $\mathrm{Rb}$ excitation linewidth broadened by Rb-He collisions. These two measurements gave the same result. The values of temperature mentioned in this paper are those of liquid helium in which the cell was placed; this temperature was almost constant even during the loading.

\section{RESULTS}

Experiments were carried out for many sample cells. Experimental results obtained for these cells were consistent qualitatively with each other, although there was a weak cell dependence. To avoid confusion, we present in this section experimental data obtained for one typical sealed cell.

Figure 2(a) shows the time evolution of the numbers of $\mathrm{Rb}$ atoms present in the cell when a loading laser beam (power $180 \mathrm{~mW}$, wavelength $720 \mathrm{~nm}$ ) is directed through the whole volume of the cell for $10 \mathrm{~s}$ at temperatures above and below $T_{c}$ (1.93 and $1.85 \mathrm{~K}$, respectively). At both temperatures, the loading begins immediately after the loading beam is turned on, and the numbers of the loaded $\mathrm{Rb}$ atoms almost reach their maximums in $10 \mathrm{~s}$. After termination of the cell loading, the gaseous atomic $\mathrm{Rb}$ resides in the cell with $1 / e$ lifetimes of about $10 \mathrm{~s}$, which are considered to be limited mainly by the movement of the $\mathrm{Rb}$ atoms to the cell walls and the subsequent loss there [6]. These lifetimes were independent of the $\mathrm{Rb}$ atomic density up to $10^{9} \mathrm{~cm}^{-3}$ we attained, indicating the $\mathrm{Rb}-\mathrm{Rb}$ two-body loss (dimer formation) was negligible. One significant difference clearly seen in Fig. 2(a) between LIAD loadings above and below $T_{c}$ is the number of $\mathrm{Rb}$ atoms loaded in $10 \mathrm{~s}$. This number, which is denoted by $N_{\mathrm{Rb}}$ in this paper, is about 20 times larger at $1.85 \mathrm{~K}$ than at $1.93 \mathrm{~K}$.

The large difference in loading efficiency can be more apparently seen in Fig. 2(b), which shows the number of $\mathrm{Rb}$

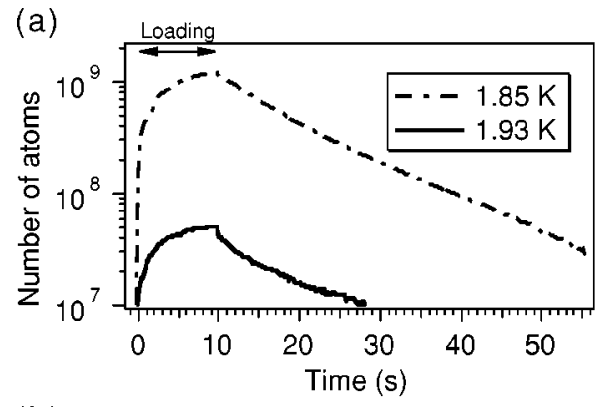

(b)

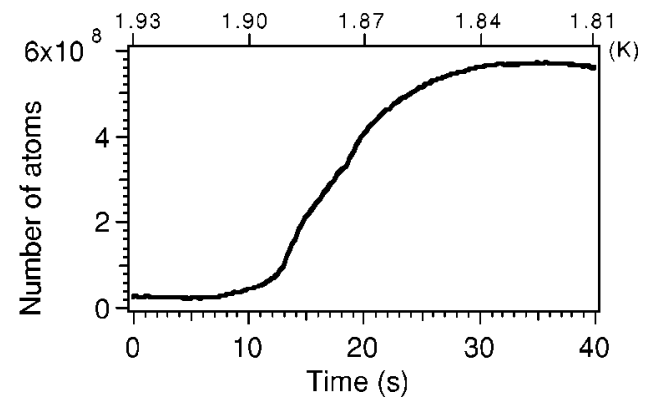

FIG. 2. (a) The time evolution of the numbers of $\mathrm{Rb}$ atoms in the cell when the cell is loaded for $10 \mathrm{~s}$ by turning on the loading beam (power $180 \mathrm{mw}$, wavelength $720 \mathrm{~nm}$ ) at $0 \mathrm{~s}$. (b) The number of $\mathrm{Rb}$ atoms present in the cell as the cell is cooled gradually by about $0.03 \mathrm{~K}$ per $10 \mathrm{~s}$ across $T_{c} \simeq 1.9 \mathrm{~K}$, while the loading beam (power $180 \mathrm{~mW}$, wavelength $720 \mathrm{~nm}$ ) is kept on. The upper scale gives the approximate temperature of the cell.

atoms present in the cell when the temperature is slowly decreased with the cell irradiated with the loading beam. The cell begins to be loaded much more effectively as the cell temperature falls across $T_{c}$, eventually being filled with a density about 20 times higher than the limiting density above $T_{c}$.

This large jump in loading efficiency was also observed at the lambda point $(2.17 \mathrm{~K})$ in the density-controllable cell containing enough helium gas that it is liquified above the lambda point. Thus we concluded that this notable change in loading efficiency is related to the superfluid transition of helium in the cell.

Figure 3 shows a series of images illustrating how the cell is loaded with $\mathrm{Rb}$ atoms below $T_{c}$ (at $1.85 \mathrm{~K}$ ). $\mathrm{Rb}$ atoms are first desorbed from the cell walls irradiated with the loading beam, and then transported into the center of the cell along with gas flows around the bottom corners of the cell, finally distributed uniformly in the cell in $10 \mathrm{~s}$. The flow speed was increased with the increase of the loading beam power. It was increased also when the temperature of the liquid helium surrounding the cell was increased gradually during the loading of the cell. Therefore the gas flow is considered to be produced by the heating of the cell. It must be noted that the direction of the gas flow is opposite to that of ordinary convection produced by heating of the walls. This observed gas flow is peculiar to the cell held below $T_{c}$, while faint but ordinary convectionlike flows that flowed up along the heated walls were observed above $T_{c}$. Therefore the strong flow shown in Fig. 3 is probably related to superfluid helium in the cell. Although investigations for a detailed understand- 


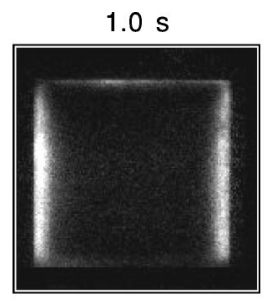

$2.4 \mathrm{~s}$
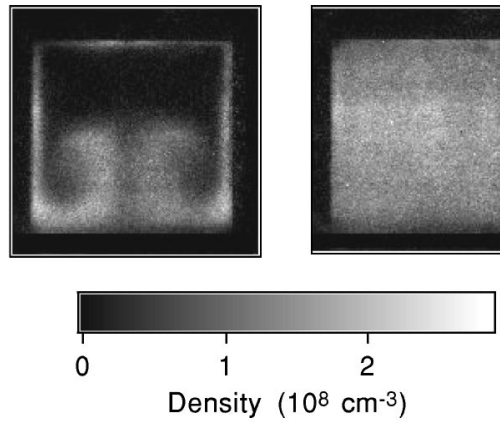

Density $\left(10^{8} \mathrm{~cm}^{-3}\right)$

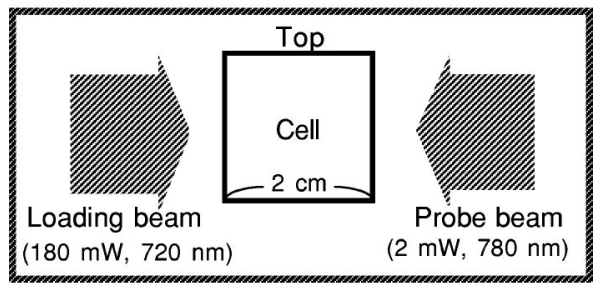

FIG. 3. Images of density distribution of $\mathrm{Rb}$ atoms loaded into the cell when the loading laser beam is turned on at $0 \mathrm{~s}$ at $1.85 \mathrm{~K}$. The ICCD camera is exposed for $0.2 \mathrm{~s}$ for one image, for example, from 0.8 to $1.0 \mathrm{~s}$ for the left-top image. Bottom: configuration of laser beams.

ing of this unique "reversed convection" are currently underway, we believe that this strong gas flow below $T_{c}$ plays an important role in the LIAD loading process, as discussed in Sec. IV B.

As stated above, LIAD loading is a simple and effective method. However, the efficiency decreases as the loading is

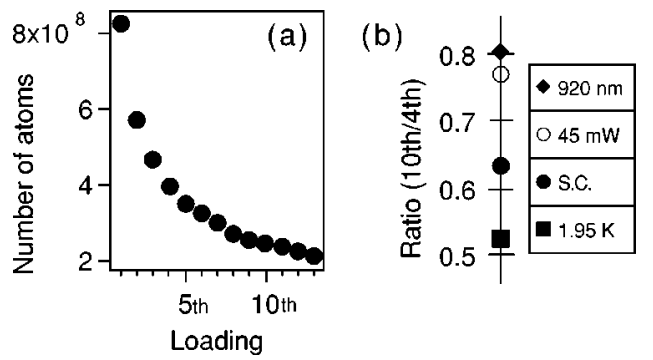

FIG. 4. (a) The decrease of the number of Rb atoms loaded in 10 $\mathrm{s}\left(N_{\mathrm{Rb}}\right)$ as 10-s loading is performed repeatedly with a loading beam of power $180 \mathrm{~mW}$ and wavelength $745 \mathrm{~mm}$ at $1.85 \mathrm{~K}$. (b) Ratios of $N_{\mathrm{Rb}}$ obtained by the tenth loading to $N_{\mathrm{Rb}}$ obtained by the fourth loading under standard conditions (power $180 \mathrm{~mW}$, wavelength $745 \mathrm{~nm}$, temperature $1.85 \mathrm{~K}$, denoted by SC). The cell is loaded five times from the fifth to the ninth loading under various conditions, whose differences from the standard conditions are noted in the figure. See Sec. IV B for details.

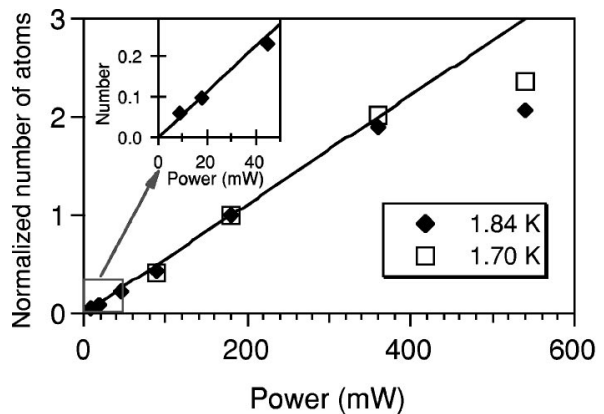

FIG. 5. The number of $\mathrm{Rb}$ atoms loaded in $10 \mathrm{~s}\left(N_{\mathrm{Rb}}\right)$ as a function of power of the loading beam, measured at 1.84 and 1.70 $\mathrm{K}$ with a loading beam of wavelength $720 \mathrm{~nm}$. Each value of $N_{\mathrm{Rb}}$ is normalized to the value for $180 \mathrm{~mW}$ at each temperature. The line shows a linear dependence on the beam power up to about $360 \mathrm{~mW}$.

performed repeatedly. Figure 4(a) displays the decrease of $N_{\mathrm{Rb}}$ as 10 -s loading is repeated with a loading beam of power $180 \mathrm{~mW}$ and wavelength $745 \mathrm{~nm}$ at $1.85 \mathrm{~K}$.

The decreased efficiency can be recovered by warming up the cell typically above about $200 \mathrm{~K}$ and cooling it again. For warming up the cell, we heated the sample chamber of the cryostat with a heater mounted inside it, or pulled up the cell to the uppermost part of the sample chamber, which was almost at room temperature. The speed of cooling the cell affected loading efficiency. Such a rapid cooling as the cell was dipped into liquid helium suddenly from the upper part of the sample chamber did not recover loading efficiency enough. We usually cooled the cell down to liquid-helium temperatures in about an hour.

The dependences of $N_{\mathrm{Rb}}$ on the power and the wavelength of the loading laser beam were investigated as well in the following way. We performed one series of measurements of $N_{\mathrm{Rb}}$ for two irradiation conditions, for example, for power $A$ and $B$ or wavelength $A$ and $B$, as applying the two conditions alternately $(A \rightarrow B \rightarrow A \rightarrow B \rightarrow \cdots)$. In a series of values measured in this way, denoted by $N_{A_{1}} \rightarrow N_{B_{1}} \rightarrow N_{A_{2}} \rightarrow N_{B_{2}}$ $\rightarrow \cdots, N_{A_{n}}$ was compared with $\left(N_{B_{n-1}}+N_{B_{n}}\right) / 2\left[N_{B_{n}}\right.$ was also compared with $\left.\left(N_{A_{n}}+N_{A_{n+1}}\right) / 2\right]$ to obtain the relative loading efficiency (for $A$ and $B$ ) that was almost free from the decrease of loading efficiency with repetitions of LIAD loading. Then we heated and cooled the cell to recover loading efficiency and carried out another series of measurements in the same way except applying the two irradiation conditions in the opposite order $(B \rightarrow A \rightarrow B \rightarrow A \rightarrow \cdots)$. We repeated the measurements with this procedure for each pair of the conditions several times and averaged the obtained values of the relative efficiency for these two conditions. Measuring these relative efficiencies over the wide ranges of the power and the wavelength of the loading beam, we obtained the results shown in Figs. 5 and 6.

Figure 5 shows the dependence of $N_{\mathrm{Rb}}$ on the power of the loading beam, normalized to the value obtained for power $180 \mathrm{~mW}$. $N_{\mathrm{Rb}}$ is approximately proportional to the power up to $360 \mathrm{~mW}$, with saturation behavior seen at higher power. One possible explanation for this saturation behavior is to ascribe the reduction of $N_{\mathrm{Rb}}$ to the heating of the cell beyond $T_{c}$ by laser irradiation. This hypothesis was not sup- 


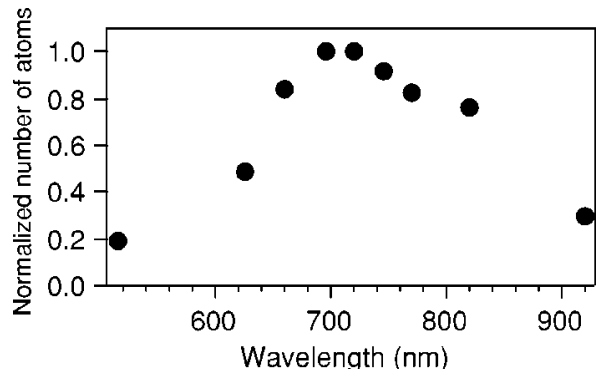

FIG. 6. The number of $\mathrm{Rb}$ atoms loaded in $10 \mathrm{~s}\left(N_{\mathrm{Rb}}\right)$ as a function of wavelength of the loading beam, measured with a loading beam of power $110 \mathrm{~mW}$ at $1.85 \mathrm{~K}$. The values of $N_{\mathrm{Rb}}$ are normalized to its peak value.

ported by our data, since saturation behavior was observed also at $1.70 \mathrm{~K}$ where the cell should not be heated beyond $T_{c}$. Note that the reduction of $N_{\mathrm{Rb}}$ was apparently observed when the cell was held just below $T_{c}$, where the cell was easily heated beyond $T_{c}$. Another possible explanation is that the loss rate that may increase with the atomic density balances with the desorption rate. This also did not seem correct, since we could achieve higher densities $(\sim 5$ $\times 10^{8} \mathrm{~cm}^{-3}$ ) of $\mathrm{Rb}$ atoms with a $180 \mathrm{~mW}$ loading beam in another cell with higher loading efficiency (see below) than the density $\left(\sim 2 \times 10^{8} \mathrm{~cm}^{-3}\right)$ at which the saturation was observed in Fig. 5. We consider that the saturation may be related to the critical rate of the helium film flow on the cell walls, and discuss this idea in Sec. IV B.

Figure 6 shows $N_{\mathrm{Rb}}$ as a function of wavelength of the loading beam. The wavelength-dependence profile peaks at about $700 \mathrm{~nm}$ and has an approximately $200 \mathrm{~nm}$ width. The profiles measured for other cells were slightly different, especially in width, although their peaks were at around 700 $\mathrm{nm}$. As discussed below, these features of wavelength dependence provide important information about the source of desorbed $\mathrm{Rb}$ atoms.

We finally describe in this section how the deposition of $\mathrm{Rb}$ on the wall surfaces during the cell preparation affects loading efficiency. The cell walls not covered visibly with $\mathrm{Rb}$ metal, as prepared in the way described in Sec. II, gave high loading efficiency. No effective loading was attained when the loading beam was directed onto the surface of visible $\mathrm{Rb}$ metal such as $\mathrm{Rb}$ droplets introduced into the cell. The Rb films on the cell walls, even though they were so thin that they could barely be recognized with the naked eye [10], were not observed to desorb atoms either. Even when the cell walls to be irradiated were not visibly covered with $\mathrm{Rb}$ metal, the introduction of $\mathrm{Rb}$ droplets into the cubic body of the cell often reduced loading efficiency. In this case the amount of $\mathrm{Rb}$ deposited on the cell surfaces probably becomes larger than in the case of no $\mathrm{Rb}$ droplets in the cell. However the walls from which $\mathrm{Rb}$ metal was very carefully removed with a torch also reduced the efficiency. Therefore a moderate amount of $\mathrm{Rb}$ on the cell surfaces seems to be required for the high efficiency of LIAD loading, although it is difficult at present to achieve the desired amount in every cell preparation since one cannot see it with the naked eye. Thus loading efficiency was different for every cell. The data presented in this section were obtained for a cell with a loading efficiency equal to about a quarter of the highest efficiency we have achieved thus far for another cell.

Another feature to be pointed out about the cell conditions is that the cells used in the experiment have lower atomic densities at room temperature than a saturated $\mathrm{Rb}$ vapor cell. In the cells used, the density distribution of the atomic Rb vapor at room temperature was not uniform and the density of $\mathrm{Rb}$ atoms near the $\mathrm{Rb}$ metal surface was higher than near the glass surface, which was observed by ICCD camera imaging. This indicates that the wall surfaces were not saturated with $\mathrm{Rb}$ and kept adsorbing (or absorbing) Rb atoms [13].

These cell conditions were changing gradually at room temperature. We stored the cells in liquid $\mathrm{N}_{2}$ when we intended to prevent the change of the cell conditions.

\section{DISCUSSION}

On the basis of the experimental results presented above, we give a consistent picture that can answer two main questions in the LIAD loading mechanism: What is the source of desorbed $\mathrm{Rb}$ atoms? Why is loading efficiency very effective below the superfluid transition temperatures?

\section{A. Source of $\mathbf{R b}$ atoms}

The dependence of $N_{\mathrm{Rb}}$ on the wavelength of the loading laser beam (Fig. 6) is an indication that $\mathrm{Rb}$ atoms are desorbed from $\mathrm{Rb}$ particles on the walls. In the past Hoheisel, Vollmer, and Träger studied extensively atom desorption by $\mathrm{cw}$ laser irradiation from alkali-metal particles $(\mathrm{Na}, \mathrm{K})$ on a substrate with mean radii of about 5-100 nm [14]. They reported that the dependence of the desorption rate on the laser wavelength exhibited a broad (full width at half maximum $\sim 100 \mathrm{~nm}$ ) resonant character, which is similar to our result. They demonstrated as well that the wavelength dependence of the desorption rate is not dependent so strongly on the particle size and is dominated mainly by the dipolar surface-plasmon resonance of the metal particle; this surfaceplasmon resonance contributes to the electric-field enhancement near the particle surface. The resonance frequency $\omega_{1}$ of the dipolar surface plasmon for a spherical metal particle is independent of the particle radius and is given by

$$
\omega_{1}=\frac{\omega_{p}}{\sqrt{1+2 \epsilon_{m}}},
$$

where $\omega_{p}$ is the volume plasma frequency of the metal and $\epsilon_{m}$ is the dielectric constant of a surrounding medium of the particle [15]. This dielectric constant $\epsilon_{m}$ can be approximated by a value between the dielectric constants of glass $(\simeq 2)$ and helium $(\simeq 1)$ for the particle on the cell wall [15]. Thus if we take $\hbar \omega_{p}=3.4 \mathrm{eV}$ ( $\hbar$ is Plank's constant divided by $2 \pi)$ [16] and $\epsilon_{m}=1.4$, we have a resonance wavelength of $710 \mathrm{~nm}$, in agreement with the wavelength at the peak of the resonance profile shown in Fig. 6.

Although it is difficult to determine the particle size from the wavelength dependence, the above "Rb-particle" picture is reliable also considering the amount of $\mathrm{Rb}$ deposited on 
the wall. $\mathrm{Rb}$ metal on the walls cannot be seen with the naked eye and therefore its thickness is less than a few tens of nanometers on the assumption that it is a continuous film [10]. In addition, the observed low $\mathrm{Rb}$ vapor density near the glass surfaces of the cell indicates that the cell surfaces are not covered completely by many layers of $\mathrm{Rb}$. Therefore $\mathrm{Rb}$ metal on the cell surfaces must exhibit a granular structure (whether discontinuous or continuous), which is typical when the amount of deposits is small [17]. Discontinuous, isolated particles of appropriate sizes, as discussed above, should provide high desorption rates, since the deposition rate is dependent on the particle size resonantly [18]. However much larger particles that may coalesce and become continuous could be a source of $\mathrm{Rb}$ atoms, although it should provide poor desorption rates. Even in that case the deposited $\mathrm{Rb}$ can be regarded as an aggregate of $\mathrm{Rb}$ particles in terms of the desorption caused by the surface-plasmon resonance because of its granular structure, and thus the " $\mathrm{Rb}$ particle" picture should be valid.

On the basis of the picture that the sources of atoms are $\mathrm{Rb}$ particles, we can interpret the observed dependences of loading efficiency on the deposition of $\mathrm{Rb}$ on the wall surfaces and on the repetitions of loading as follows: It is important, for achieving high LIAD loading efficiency, that $\mathrm{Rb}$ metal is distributed as particles of appropriate sizes on the surfaces at low temperatures. The more (or less) $\mathrm{Rb}$ is deposited on the surfaces when the cell is prepared, the larger (or smaller) the particles become [14], resulting in poor loading efficiency. Visible $\mathrm{Rb}$ films, which are formed by a far larger amount of $\mathrm{Rb}$ deposits, are not suitable either [19]. The speed of the cell cooling could affect the particle conditions such as the size distribution and the number of the desorption sites such as surface defects or roughnesses [14] from which $\mathrm{Rb}$ atoms can be desorbed, although a detailed model to explain the experimental result on the dependence of the efficiency on the cooling speed is not clear at present. The decrease of loading efficiency with the repetitions of loading, which was also reported in Ref. [14], is due to the changes of the $\mathrm{Rb}$ particle conditions such as the decrease of the number of the surface defects or roughnesses. The particle conditions suitable for LIAD loading are not recovered (at least quickly) at liquid-helium temperatures, which is indicated by the fact that the decreased loading efficiency was not recovered by holding the cell at liquid-helium temperatures for as long as $2 \mathrm{~h}$, during which desorbed $\mathrm{Rb}$ atoms should be adsorbed on the walls. Heating the cell and cooling it down again cause diffusion and reformation of $\mathrm{Rb}$ atoms and particles on the cell surfaces [12], and consequently recover the original conditions (and hence the desorption rate).

It must be noted that the observed LIAD at liquid-helium temperatures is surprising since the exponential reduction of the desorption rate with decreasing temperature from room temperature down to about $150 \mathrm{~K}$ has been observed [14,21]. LIAD at liquid-helium temperatures may be explained by a weak dependence of the desorption rate on temperature in the low-temperature range as already seen in Ref. [14] at about $100 \mathrm{~K}$, where the desorption rate is estimated to be roughly of the same order as our measured rates if the same laser power and particle density are assumed. However in our measurements at temperatures up to $250 \mathrm{~K}$ a strong (exponential) temperature dependence was not observed. We do not have a clear idea at present to explain these results and further investigations would be valuable.

\section{B. Role of superfluidity}

We propose that the role of superfluidity in the great increase of loading efficiency below the superfluid transition temperatures is an efficient transport of desorbed atoms into the cell by superfluid-driven gas flows, which are shown in Fig. 3.

We consider the transport process as follows. Without the gas flows, atoms desorbed from the surfaces of the walls can hardly diffuse into the cell volume because of their slow diffusion in the dense helium gas, then eventually recondense on the walls [22]. However, heating the cell walls covered with a superfluid helium film with laser beams causes the continuous evaporation of the helium film, which is supplied by the superfluid flow on the walls from the bulk liquid helium at the bottom of the cell. This evaporation produces helium gas flows, which can transport $\mathrm{Rb}$ atoms into the dense helium gas. Note that the amount of vaporized helium in the cell is about $3 \times 10^{19}$ atoms if the cell is heated during a typical loading from 1.85 to $1.87 \mathrm{~K}$. This amount is much larger than that of desorbed $\mathrm{Rb}$ atoms and is large enough to produce gas flows carrying the $\mathrm{Rb}$ atoms in the dense helium gas with a density of about $8 \times 10^{19} \mathrm{~cm}^{-3}$ at $1.85 \mathrm{~K}$.

To verify this picture, we consider an extreme case that $\mathrm{Rb}$ atoms are introduced into the cell only by diffusion. This should well model the loading process above $T_{c}$. In this extreme case, $\mathrm{Rb}$ atoms desorbed from the walls are thermalized by collisions with helium atoms. The average temperature change $\Delta T$ of the desorbed atoms per collision with helium atoms is given, for elastic hard-sphere collisions, by

$$
\Delta T=-\frac{8}{3} \frac{m M}{(m+M)^{2}}\left(T-T_{\mathrm{He}}\right),
$$

where $M$ and $m$ are the masses of $\mathrm{Rb}$ and $\mathrm{He}$ atoms, respectively, and $T$ and $T_{\mathrm{He}}$ are their temperatures [25]. Accordingly, if we assume that the initial temperature of the $\mathrm{Rb}$ atoms is about $10^{3} \mathrm{~K}$ [14], they require about $10^{2}$ collisions with helium atoms of $2 \mathrm{~K}$ to be thermalized. Taking an elastic-collision cross section for Rb-He to be $10^{-14} \mathrm{~cm}^{2}$ and therefore the mean free path of $\mathrm{Rb}$ atoms to be $10^{-6} \mathrm{~cm}$ in helium gas with a density of $10^{20} \mathrm{~cm}^{-3}$, we can estimate that the desorbed atoms are detached away from the surface of the wall by at most $10^{-4} \mathrm{~cm}$ before being thermalized.

These thermalized $\mathrm{Rb}$ atoms then diffuse in the cell. To estimate how many $\mathrm{Rb}$ atoms are introduced into the cell volume by diffusion, we carried out an analytical calculation of diffusion in a cubic cell (2-cm sides). We assumed that $\mathrm{Rb}$ atoms colliding with the surfaces were condensed, namely, a zero atomic density at the boundaries. Atom desorption from one wall and subsequent thermalization by collisions with helium gas within a distance $d$ from the wall was approximated by a plane source (at a distance $d$ from the wall) from 

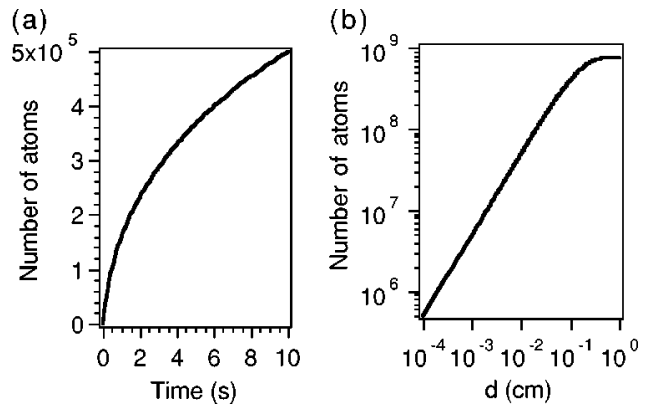

FIG. 7. (a) The calculated number of atoms in a cubic cell $(2-\mathrm{cm}$ sides). The atoms diffuse into the cell from a plane source from which atoms are liberated continuously at a rate of $2.5 \times 10^{7}$ atoms $/\left(\mathrm{cm}^{2} \mathrm{~s}\right)$. This plain source is positioned at a distance $d=10^{-4} \mathrm{~cm}$ from one surface. A zero atomic density at the boundaries is assumed. The diffusion coefficient is 3 $\times 10^{-3} \mathrm{~cm}^{2} / \mathrm{s}$. (b) The number of atoms present in the cell at $10 \mathrm{~s}$ as a function of $d$.

which atoms were liberated continuously at an assumed rate of $2.5 \times 10^{7}$ atoms $/\left(\mathrm{cm}^{2} \mathrm{~s}\right)$. The diffusion coefficient was taken to be $3 \times 10^{-3} \mathrm{~cm}^{2} / \mathrm{s}$, which had been calculated for $\mathrm{Rb}$ atoms in the saturated helium gas at $1.85 \mathrm{~K}$ [6]. Figure 7(a) shows the time evolution of the number of atoms present in the cell, calculated for $d=10^{-4} \mathrm{~cm}$. We see that only less than a thousandth of the produced atoms are loaded into the cell, while the other atoms are lost on the wall. Therefore it is concluded that the desorbed atoms are hardly loaded into the cell only by diffusion without support of the helium-gas flow. However, if the desorbed atoms are detached farther away from the walls by the superfluid-driven gas flow below $T_{c}$, loading efficiency improves. This can be seen in Fig. 7(b), where the increase of $N_{\mathrm{Rb}}$ with increasing $d$ is displayed. Thus it is shown that the helium-gas flow produced by the evaporation of the superfluid film can dramatically increase loading efficiency.

To verify our model further from a different point of view, we experimentally examine the picture that the loading efficiency difference at temperatures below and above the superfluid transition temperature is not associated with a change in the desorption rate. For this purpose, the decrease of $N_{\mathrm{Rb}}$ with the repetitions of loading under various conditions was measured [see Fig. 4(b)]. In the same way as the measurements shown in Fig. 4(a), we performed LIAD loading four times under standard conditions [beam power $180 \mathrm{~mW}$, wavelength $745 \mathrm{~nm}$, temperature $1.85 \mathrm{~K}$, denoted by SC in Fig. 4(d)] and recorded $N_{\mathrm{Rb}}$ obtained by the last (namely, the fourth) loading. We next loaded the cell five times under one of the various conditions, which were different in power or wavelength of the loading beam or in cell temperature from the standard conditions. These differences are noted in Fig. 4(b). Then we loaded the cell again under the standard conditions (the tenth loading) and recorded $N_{\mathrm{Rb}}$. The two values of $N_{\mathrm{Rb}}$ obtained by the fourth and the tenth loading, namely, measured before and after the cell was loaded five times under one of the various conditions, were compared to show the decrease of $N_{\mathrm{Rb}}$ under the various conditions.

These data can be exploited to confirm our model. The decrease of $N_{\mathrm{Rb}}$ with the repetitions of loading indicates, as mentioned in Sec. IV A, that the desorption rate of $\mathrm{Rb}$ atoms decreases as $\mathrm{Rb}$ particles on the walls release $\mathrm{Rb}$ atoms. Therefore the decrease rate of $N_{\mathrm{Rb}}$ should depend not on the amount of the loaded atoms but on that of the desorbed atoms. This is confirmed qualitatively by experimental data taken at $1.85 \mathrm{~K}$ as follows: The beam wavelength $(920 \mathrm{~nm})$ far away from the resonance center $(700 \mathrm{~nm})$ and the low beam power $(45 \mathrm{~mW})$ compared to the standard conditions (745 nm, $180 \mathrm{~mW}$ ) give small $N_{\mathrm{Rb}}$ as shown in Figs. 5 and 6, and this low loading efficiency can be ascribed to the small desorption rate of Rb atoms (see Sec. IV A and the discussion about the power dependence below). In these two cases, the decrease rates of $N_{\mathrm{Rb}}$ are smaller than under the standard conditions as shown in Fig. 4(b).

On the other hand, above $T_{c}$ (at $1.95 \mathrm{~K}$ ), although $N_{\mathrm{Rb}}$ is about 20 times smaller than at $1.85 \mathrm{~K}$ as shown in Fig. 2, the decrease rate of loading efficiency is larger than at $1.85 \mathrm{~K}$. This means that the desorption rate at $1.95 \mathrm{~K}$ is larger than at $1.85 \mathrm{~K}$, which is very contrary to the reduction of LIAD loading efficiency by a factor of 20 at $1.95 \mathrm{~K}$. Although the reason why the desorption rate is higher at $1.95 \mathrm{~K}$ is not clear at present, this result indicates that the increase of loading efficiency below the superfluid transition temperatures is not due to the increase of the atom desorption rate, in agreement with our model.

We finally interpret the dependence of $N_{\mathrm{Rb}}$ on the loading beam power (Fig. 5) on the basis of our picture of the loading mechanism. Assuming a linear dependence of the desorption rate on the loading beam power, as demonstrated over four orders of magnitude of power in Ref. [14], the linear increase of $N_{\mathrm{Rb}}$ in the power range of 9-360 $\mathrm{mW}$ depends mainly on the increase of the desorption rate. This implies that transport efficiency is independent of the beam power in this power range, although the evaporation rate of the helium film should increase with increasing beam power until it reaches the critical rate determined by the critical flow rate of the film from the bulk liquid helium. One possible explanation is that transport efficiency is saturated already for the evaporation rates in this power range. If the beam power exceeds a critical power that makes the film flow rate critical, the cell walls become dry because the film flowing up the walls is completely evaporated before it covers the whole surface of the walls. This can lead to the reduction in transport efficiency and therefore the saturation of $N_{\mathrm{Rb}}$ shown in Fig. 5.

To verify this interpretation of the saturation, we make some evaluations as follows. Taking a critical film flow rate over a perimeter of length $4 \mathrm{~cm}$ [the side length of the cell (2 $\mathrm{cm}$ ) times the number of the irradiated walls (two)] to be 3 $\times 10^{-4} \mathrm{~cm}^{3} / \mathrm{s}\left(7 \times 10^{18}\right.$ atoms/s $)$ [7], we can estimate the power necessary to completely evaporate the film flowing up the two side walls at the critical rate to be about $1 \mathrm{~mW}$ [7]. This power is about a few parts per thousand of the beam power at which the saturation of $N_{\mathrm{Rb}}$ was observed, reasonable in view of the absorbance of the glass cell walls for the laser beam [26].

The above explanation of the power dependence seems reasonable, but would need further examinations by a more precise characterization for the transport process, which will be the subject of future investigations. 


\section{CONCLUDING REMARKS}

In this paper we have presented extensive studies on LIAD loading of $\mathrm{Rb}$ atoms into cold helium-gas cells. On the basis of our experimental results, we have given a consistent picture of the LIAD loading mechanism: Rb atoms are optically desorbed from $\mathrm{Rb}$ particles formed on the cell walls and then transported into the cell by superfluid-driven gas flows. The resonant profile of the wavelength dependence of loading efficiency (Fig. 6) strongly supports this picture of LIAD. The decrease of loading efficiency with the repetitions of loading (Fig. 4), its recovery by heating and cooling the cell, and the dependence of loading efficiency on the amount of $\mathrm{Rb}$ deposited on the walls are also consistent with this picture of LIAD considering the results of the past studies on LIAD from alkali-metal particles in Ref. [14]. The picture of the transport by the superfluid-driven gas flows was examined both theoretically (Fig. 7) and experimentally [Fig. 4(b)], and turned out to be fairly reliable. However, in the future, more quantitative studies should be performed. For example, preparing the sample of $\mathrm{Rb}$ particles with its size distribution controlled on clean substrates in ultrahigh vacuum should increase our understanding of the desorption mechanism at liquid-helium temperatures. Also, a more quantitative characterization for the superfluid-driven gas flow (Fig. 3) and the transport process, which are key to examine the tentative explanation of the power dependence (Fig. 5), would be important. These investigations should reveal how to obtain high atomic $\mathrm{Rb}$ densities, which are very useful for future applications of the cold cells.

Here we mention preliminary results of our attempts to load other alkali-metal atoms (K,Cs) by LIAD. We have loaded a cell with $\mathrm{K}$ atoms successfully and obtained results almost consistent with the case of $\mathrm{Rb}$. However, it is worth noting the wavelength dependence of loading efficiency for $\mathrm{K}$. Its resonant profile was blueshifted $(\sim-10 \mathrm{~nm})$ compared to $\mathrm{Rb}$, but not so distinguishable from $\mathrm{Rb}$ as expected from the volume-plasmon resonance for $K$ [16], which is at a much shorter wavelength $(\sim-50 \mathrm{~nm})$ than for $\mathrm{Rb}$. The expected difference between $\mathrm{Rb}$ and $\mathrm{K}$ was probably obscured by other conditions such as the particle-size distribution [14]. For $\mathrm{Cs}$, on the other hand, we have not succeeded in LIAD loading yet, reasons for which are still under investigation.

Finally, we comment on the conventional laser-ablationloading method [28] we also attempted. An ablative loading, namely, irradiating the metal surface or the cell wall covered slightly with metal with a focused, high peak-power pulsed laser, could fill the cell with metal vapors. This "violent" method was less dependent on conditions such as cell temperature, sample metal species, and loading repetitions than the "gentle" LIAD loading method. It was especially useful to obtain a temporary high atomic density. However one disadvantage was that the properties of the produced vapors, for example, the lifetime of gaseous atoms, were not reproducible; it seems to depend on the conditions of the tiny surface area on which the pulsed laser was focused. For different positions on the surface on which the beam was focused, the lifetimes of loaded atoms changed in the range between less than $1 \mathrm{~s}$ and almost $10 \mathrm{~s}$ even for the same density of atoms loaded into the cell. This fluctuation may be caused by produced metal clusters, which can adsorb $\mathrm{Rb}$ atoms in the helium gas and reduce their lifetimes; some of these clusters could actually be observed even by watching the scattered light from them with the naked eye when the laser ablation loading was performed.

\section{ACKNOWLEDGMENTS}

We would like to thank A. R. Young and M. Kumakura for their useful comments and critical readings of the manuscript. This work was supported by Grants-in-Aid for Scientific Research from the Ministry of Education, Science and Culture of Japan (Grants Nos. 11304023 and 11216203) and the Japan-U.S. cooperative science program of the Japan Society for the Promotion of Science (JSPS). One of the authors (A.H.) acknowledges support from JSPS.
[1] As reviews of studies on species in bulk helium, see M. Takami, Comments At. Mol. Phys. 32, 219 (1996); B. Tabbert, H. Günther, and G. zu Putlitz, J. Low Temp. Phys. 109, 653 (1997); S. I. Kanorsky and A. Weis, Adv. At., Mol., Opt. Phys. 38, 87 (1997); E. B. Gordon and A. F. Shestakov, Fiz. Nizk. Temp. 26, 5 (2000) [Low Temp. Phys. 26, 1 (2000)]. On helium-cluster-isolation spectroscopy, see J. P. Toennies and A. F. Vilesov, Annu. Rev. Phys. Chem. 49, 1 (1998); Y. Kwon, P. Huang, M. V. Patel, D. Blume, and K. B. Whaley, J. Chem. Phys. 113, 6469 (2000). On helium-buffer-gas loading, see R. deCarvalho, J. M. Doyle, B. Friedrich, T. Guillet, J. Kim, D. Patterson, and J. D. Weinstein, Eur. Phys. J. D 7, 289 (1999).

[2] J. Kim, B. Friedrich, D. P. Katz, D. Patterson, J. D. Weinstein, R. DeCarvalho, and J. M. Doyle, Phys. Rev. Lett. 78, 3665 (1997).

[3] M. Hartmann, R. E. Miller, J. P. Toennies, and A. Vilesov, Phys. Rev. Lett. 75, 1566 (1995).

[4] S. Grebenev, J. P. Toennies, and A. F. Vilesov, Science 279,
2083 (1998).

[5] T. Kinoshita, Y. Takahashi, and T. Yabuzaki, Phys. Rev. B 49, 3648 (1994); M. Arndt, S. I. Kanorsky, A. Weis, and T. W. Hänsch, Phys. Rev. Lett. 74, 1359 (1995); J. Higgins, W. E. Ernst, C. Callegari, J. Reho, K. K. Lehmann, G. Scoles, and M. Gutowski, ibid. 77, 4532 (1996); S. Lang, S. Kanorsky, T. Eichler, R. Müller-Siebert, T. W. Hänsch, and A. Weis, Phys. Rev. A 60, 3867 (1999).

[6] A. Hatakeyama, K. Oe, K. Ota, S. Hara, J. Arai, T. Yabuzaki, and A. R. Young, Phys. Rev. Lett. 84, 1407 (2000).

[7] J. Wilks, The Properties of Liquid and Solid Helium (Clarendon, Oxford, 1967).

[8] CRC Handbook of Chemistry and Physics, 74th ed., edited by David R. Lide (CRC, Boca Raton, FL, 1993), pp. 4-124.

[9] All laser beams used in the experiment were expanded to entirely cover the cell, and therefore their intensities expressed in power $/ \mathrm{cm}^{2}$ were given approximately by their powers divided by 4 . 
[10] Rb films of as thin as $30 \mathrm{~nm}$ thickness can be recognized with the naked eye, since their transmittance for normal incident visible light of wavelength $550 \mathrm{~nm}$ is about $80 \%$ [11], estimated using the refraction index reported in Ref. [12].

[11] O. S. Heavens, Optical Properties of Thin Solid Films (Butterworths, London, 1955).

[12] J. Monin and G.-A. Boutry, Phys. Rev. B 9, 1309 (1974).

[13] A. Gallagher and E. L. Lewis, J. Opt. Soc. Am. 63, 864 (1973).

[14] W. Hoheisel, M. Vollmer, and F. Träger, Phys. Rev. B 48, 17463 (1993).

[15] U. Kreibig and M. Vollmer, Optical Properties of Metal Clusters (Springer-Verlag, Berlin, 1995).

[16] H. Raether, Excitation of Plasmons and Interband Transitions by Electrons, Springer Tracts in Modern Physics Vol. 88 (Springer-Verlag, Berlin, 1980).

[17] M. Rasigni, G. Rasigni, J. P. Gasparini, and R. Fraisse, J. Appl. Phys. 47, 1757 (1976).

[18] The size dependence of the desorption rate peaks at a radius of about $50 \mathrm{~nm}$ for K particles according to Ref. [14].

[19] For thin metal films, desorption by the attenuated-totalreflection method might be effective [20].

[20] I. Lee, J. E. Parks II, T. A. Callcott, and E. T. Arakawa, Phys. Rev. B 39, 8012 (1989).

[21] A. M. Bonch-Bruevich, Yu. N. Maksimov, S. G. Przhibel'skii, and V. V. Khromov, Zh. Eksp. Teor. Fiz. 92, 285 (1987) Sov. Phys. JETP 65, 161 (1987).

[22] A helium film on the cell walls may reduce the loss rate of $\mathrm{Rb}$ atoms on the walls due to the small binding energy of $\mathrm{Rb}$ atoms to the surface of liquid helium [23]. However, the slow diffusion of $\mathrm{Rb}$ atoms in the dense helium gas should obscure this effect because once they approach the walls, they can hardly leave the vicinity of the cell surfaces [24]. We have not obtained experimental results suggesting the reduction of the surface loss of $\mathrm{Rb}$ atoms [6].

[23] F. Ancilotto, E. Cheng, M. W. Cole, and F. Toigo, Z. Phys. B: Condens. Matter 98, 323 (1995).

[24] F. Masnou-Seeuws and M.-A. Bouchiat, J. Phys. (France) 28, 406 (1967).

[25] A. M. Cravath, Phys. Rev. 36, 248 (1930).

[26] In the cell-heating process, most of the heat is expended by the evaporation of helium, because the heat capacities of gaseous and liquid helium in the cell and the glass cell itself are small $[7,27]$.

[27] G. K. White, Experimental Techniques in Low-Temperature Physics (Clarendon, Oxford, 1987).

[28] See, for example, Y. Matsuo, T. Nakajima, T. Kobayashi, and M. Takami, Phys. Rev. A 59, 2071 (1999). 